\title{
Differential cellular gene expression in duck trachea infected with a highly or low pathogenic H5N1 avian influenza virus
}

Pascale Massin ${ }^{1,3^{*}}$, Claire Deleage ${ }^{1,3}$, Aurélie Oger ${ }^{1,3}$, François-Xavier Briand $^{1,3}$, Hélène Quenault ${ }^{2,3}$ and Yannick Blanchard ${ }^{2,3}$

\begin{abstract}
Background: Avian influenza A (Al) viruses of subtypes $\mathrm{H} 5$ can cause serious disease outbreaks in poultry including panzootic due to H5N1 highly pathogenic (HP) viruses. These viruses are a threat not only for animal health but also public health due to their zoonotic potential. The domestic duck plays a major role in the epidemiological cycle of influenza virus subtypes $\mathrm{H} 5$ but little is known concerning host/pathogen interactions during influenza infection in duck species. In this study, a subtracted library from duck trachea (a primary site of influenza virus infection) was constructed to analyse and compare the host response after a highly or low pathogenic (LP) H5N1-infection.

Results: Here, we show that more than 200 different genes were differentially expressed in infected duck trachea to a significant degree. In addition, significant differentially expressed genes between LPAl- and HPAl-infected tracheas were observed. Gene ontology annotation was used and specific signalling pathways were identified. These pathways were different for LPAI and HPAl-infected tracheas, except for the CXCR4 signalling pathway which is implicated in immune response. A different modulation of genes in the CXCR4 signalling pathway and TRIM33 was induced in duck tracheas infected with a HPAl- or a LPAl-H5N1.

Conclusion: First, this study indicates that Suppressive Subtractive Hybridization (SSH) is an alternative approach to gain insights into the pathogenesis of influenza infection in ducks. Secondly, the results indicate that cellular gene expression in the duck trachea was differently modulated after infection with a LPAl-H5N1 or after infection with a HPAI-H5N1 virus. Such difference found in infected trachea, a primary infection site, could precede continuation of infection and could explain appearance of respiratory symptoms or not.
\end{abstract}

Keywords: Avian influenza virus, Highly pathogenic influenza, Low pathogenic influenza, H5N1, Suppressive subtraction hybridisation, Microarray, Muscovy duck, Trachea, Host-pathogen interactions

\section{Background}

The Influenza A virus genus is divided into subtypes based on the combination of two surface glycoproteins: hemagglutinin (HA, 16 subtypes) and neuraminidase (NA, 9 subtypes) [1]. The subtypes $\mathrm{H} 5$ and $\mathrm{H} 7$ of avian influenza A (AI) viruses can be both further divided into two groups of high or low pathogenic influenza A viruses (HPAI or LPAI, respectively) [2]. LPAI viruses

\footnotetext{
* Correspondence: pascale.massin@anses.fr

'Anses Ploufragan/Plouzané laboratory, Virologie Immunologie Parasitologie Aviaires et Cunicoles, B.P. 53, Ploufragan 22440, France

${ }^{3}$ European University of Brittany, Rennes, France

Full list of author information is available at the end of the article
}

induce mild or no symptoms in domestic ducks but replicate massively into the intestinal tract allowing the release of high titres of virus into faeces. In contrast, H5-HPAI viruses induce various clinical signs ranging from asymptomatic respiratory and digestive tract infections to systemic and severe symptoms leading to fatal outcome depending on age and duck species [3]. AI viruses have caused several serious epizootics within poultry, in particular the HPAI H5N1 virus which propagated between 2003 and 2006 from Asia to Europe and Africa, and caused the death of millions of chickens and other poultry leading to massive economic loss

\section{Biomed Central}

(c) 2013 Massin et al.; licensee BioMed Central Ltd. This is an Open Access article distributed under the terms of the Creative Commons Attribution License (http://creativecommons.org/licenses/by/2.0), which permits unrestricted use, distribution, and reproduction in any medium, provided the original work is properly cited. 
$[4,5]$. Indeed, poultries are key intermediates in the transmission of AI from wild avian species to mammalian species such as pigs and humans. The $\mathrm{H} 5$ virus is now enzootic in several Asian countries and represents one major concern for animal health but also for public health due to its zoonotic and pandemic potential. Indeed HPAI $\mathrm{H} 5 \mathrm{~N} 1$ has been transmitted from poultry to humans and was responsible for the death of 374 people as reported by WHO the 26th April 2013 [6,7].

Various studies have been performed to better characterize host/pathogen interactions between mammals and influenza A viruses and to identify key genes or pathways implicated in the virus pathogenicity and host response to infection [8-13]. Virus-host interaction investigations have been particularly boosted by the development of the microarray technology in many mammalian models [14-20], for which microarray tools, gene annotation and signalling pathways description are abundant. In contrast information concerning the molecular pathogenesis of AIV and the regulation of host gene response after AIV infection in avian species is scarce and essentially performed in chickens. The recent release of the chicken genome is a great help for scientists to investigate mechanisms involved in host response to the infection in chicken and the appropriate microarrays have been developed recently and used in various virus infection studies [21-30]. Concerning duck species, no microarrays are available and molecular genetics tools have made their first steps recently [31].

Some studies have described the difference of pathobiology between a LPAI and a HPAI infection in various ducks species but to our knowledge, only three studies have compared the response of ducks following LPAI or HPAI infection, focusing on immune response only [32-34]. In these studies, the authors concluded that a difference of immune response, specific for the virus and the infected tissue (lung or intestine), might explain the difference of pathogenicity between LPAI and HPAI infection in Pekin ducks. A few other studies have been performed to compare the immune response of Pekin, Mallard and Muscovy ducks and provide evidences of some differences that might account for the variability of HPAI pathogenesis in between duck species [34-37]. A limitation of these comparative studies is their focus on a very limited number of genes, usually selected on the basis of published data in other species for which the symptoms and outcome of an AI infection might be different from those observed in duck species. Therefore, these studies provide a very limited amount of information which is not necessarily the most relevant for a duck model, and thus does not give an objective overview of host/pathogen interactions between duck, cells or tissues, and influenza A virus.
For a better understanding of the host/pathogen interactions between $\mathrm{H} 5 \mathrm{~N} 1$ viruses and domestic ducks and with the aim of giving an overview of host/pathogen interactions between duck respiratory tract and HP- as compared to LP-influenza A virus, we examined and compared the mRNA expression of genes into a primary infection site, the trachea, after infection with a HPAI or a LPAI H5N1. To overcome the fact that there was no release of the duck genome and no duck specific microarray, we focused on only differentially expressed genes by creating subtracted libraries from duck trachea and used them to set up a duck microarray for analysis of HPAI and LPAI H5N1 infection.

\section{Results}

\section{Validation of the experimental infection model}

Tracheal explants were prepared as described in Materials and methods and infected with $200 \mu \mathrm{l}$ of $2 \times 10^{7}$ $\mathrm{TCID}_{50} / \mathrm{ml}$ of HPAI virus per trachea, the same dose as the one used for library construction. At $24 \mathrm{~h}$ p.i., the ciliary beats were dramatically reduced to up to $30 \%$ as compared to mock-infected tracheas (100\% ciliary beats) attesting for an efficient infection of the tracheas. To verify that infection occurred all along tracheas at $24 \mathrm{~h}$ p.i., LPAI-infected tracheas were cut into 3 parts and subjected to RNA extraction and to amplification of influenza matrix (M) segment using real-time RT-PCR as described in Materials and methods. Levels of copy numbers were similar for the three parts of trachea at $24 \mathrm{~h}$ p.i. ( 4 to $8 \cdot 10^{7} \mathrm{M}$ copy per $\mu \mathrm{l}$ ) assessing for a homogeneous contact of the inoculum with the whole trachea. In addition, RNA of LPAI- and HPAI-infected tracheas used for the preparation of probes were also subjected to amplification of influenza matrix $M$ segment using real-time RT-PCR and we did not observe significant difference.

\section{Construction of specific duck trachea subtracted libraries}

Four subtracted libraries were constructed using entire tracheal explants from SPF Muscovy ducks, infected or not with a French HPAI H5N1 strain belonging to clade 2.2.1 and subjected to the subtractive suppression hybridisation ( $\mathrm{SSH}$ ) procedure: two viral-induced and two viral-repressed cDNA libraries corresponding to sequences induced and repressed, respectively, at $4 \mathrm{~h}$ or $8 \mathrm{~h}$ p.i. From these 4 libraries, 1141 individual bacterial clones were randomly isolated for the two viral-induced libraries and 950 bacterial clones for the two viralrepressed libraries representing around $10 \%$ of all clones obtained. Those 2091 clones were used to construct our duck specific microarray as described in the Materials and methods section.

The 2091 clones were subjected to sequencing as described in the Materials and methods section. For 1013 
sequences out of 2091, a relevant blast result was obtained. For the 1078 remaining sequences we did not obtain a significant blast result due to either poor sequence quality or the absence of positive match in Genbank library. The e-values distribution of relevant blast results shown that $52 \%$ of sequences were above $10^{-140}$, supporting an accurate identification of the genes (Figure 1). 114 sequences had good homology with avian mitochondrial genes and 115 sequences had homology with avian BAC clones or complete avian cDNA of putative protein of unknown function. 784 sequences have been clearly identified, to date, as avian genes and corresponding to 210 different genes. These genes belong to several different functional families: cell cycle, metabolism, immune response, cytoskeleton network.

\section{Statistical analysis of microarray results}

In order to compare the HPAI and LPAI infections in the trachea using our microarray, new tracheal explants were infected with either strains (HPAI or LPAI) and RNA were extracted and processed for microarray experiment as described in Materials and methods section. Sixteen microarrays were hybridised allowing four replicates for each experimental condition: 4 microarrays for each time point of the experiment ( 4 and $8 \mathrm{~h}$ p.i.) and for each strain (LPAI and HPAI).

After normalisation of the raw data, we performed a first statistical analysis by comparing signals obtained with probes corresponding to infected tracheas (HPAIor LPAI-infected) versus signals obtained with probes corresponding to mock-infected tracheas. For this purpose, SAM software was used and results were considered for a calculated false discovery rate (FDR) of $5 \%$. SAM plot results are presented in Figure 2A and B. At
4 h p.i., 49 spots were up-regulated and 148 spots were down-regulated in LPAI-infected tracheas whereas 36 spots were up-regulated but 0 down regulated genes in HPAI-infected tracheas (Figure 2A and B, left panel). At $8 \mathrm{~h}$ p.i., number of differentially expressed sequences significantly increased (Figure $2 \mathrm{~A}$ and $\mathrm{B}$, right panel). In LPAI-infected tracheas, 368 sequences were up-regulated and 493 sequences were down-regulated. In HPAIinfected tracheas, 175 sequences were up-regulated and 222 were down-regulated.

This first SAM analysis gave us the difference in between infected samples compared to mock-infected ones. In a second approach, we performed a statistical analysis comparing the signals obtained with probes from HPAI-infected tracheas to signals obtained with probes from LPAI-infected tracheas. Such a comparison was possible as the reference sample used for the baseline was generated with the same pool of mock-infected duck mRNA. Results are presented in Figure 2C. A FDR fixed around $5 \%$ gave a very low number of significant differentially expressed spots between HPAI and LPAI samples: 8 down-regulated and 5 up-regulated at $4 \mathrm{~h}$ p.i. and 65 down-regulated and 19 up-regulated at $8 \mathrm{~h}$ p.i. for HPAI as compared to LPAI.

\section{Functional characterisation of differentially expressed genes}

In order to further characterize differentially expressed sequences, sequencing data and microarray results were cross-analysed using Gene Ontology annotations with the Ingenuity Pathway Analysis software. Due to the poverty of bibliographic information for the chicken annotated genome and the duck genome, comparison was made by analogy to the orthologous genes annotation

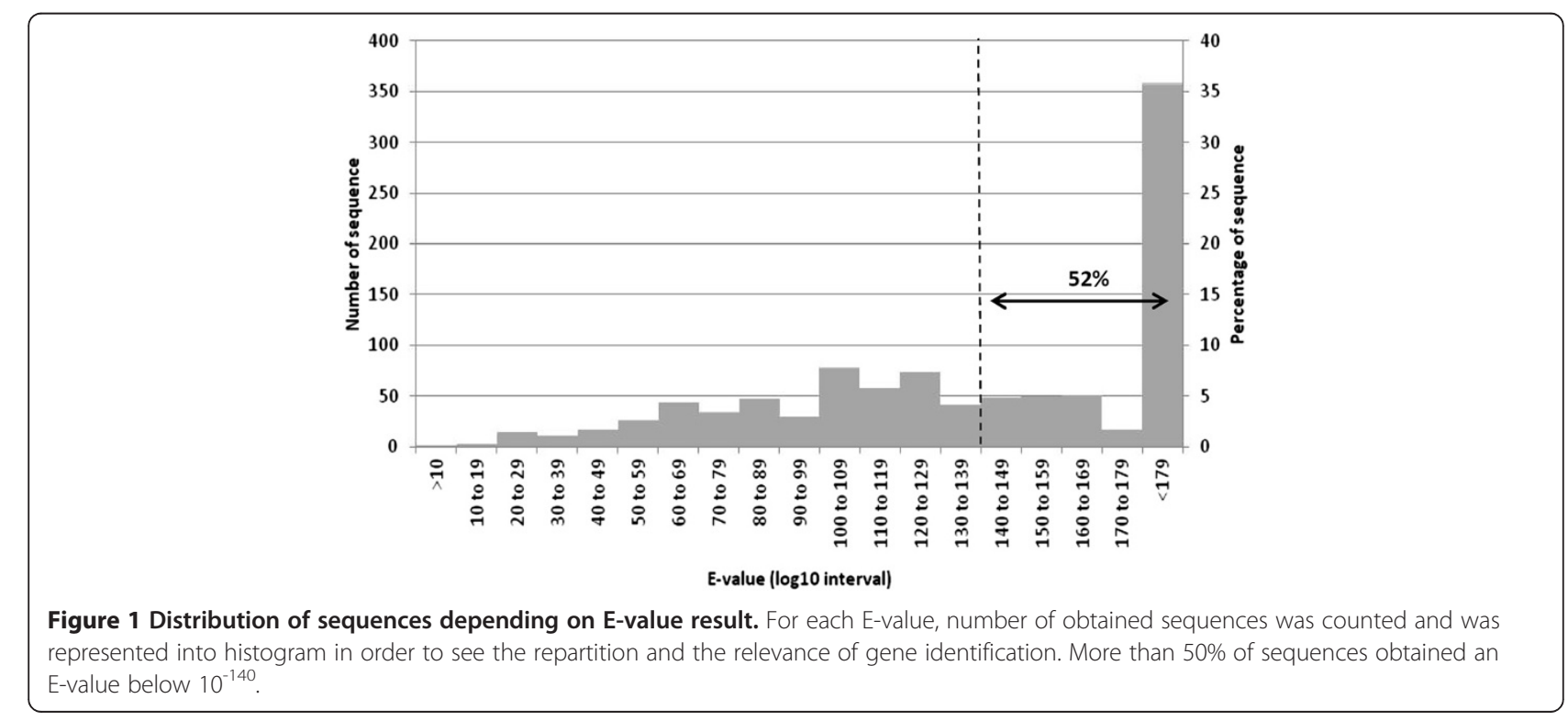



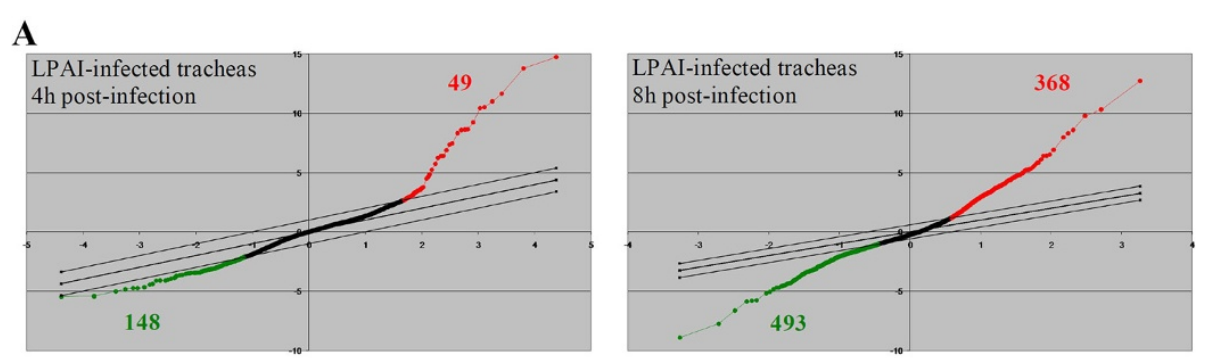

\section{B}
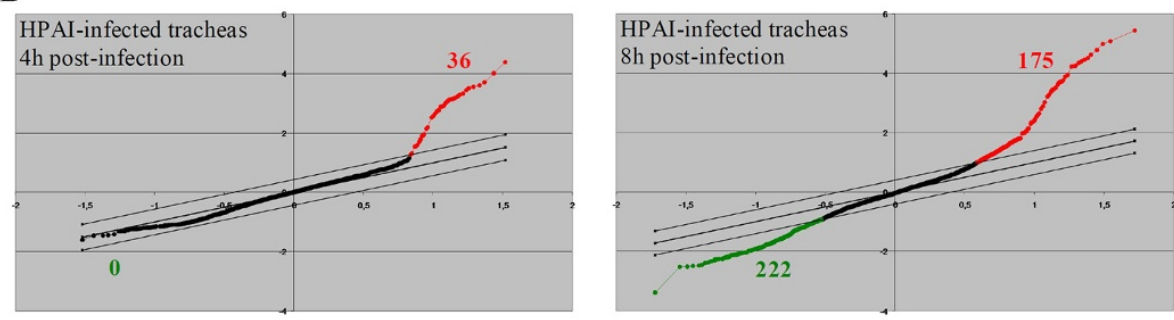

C
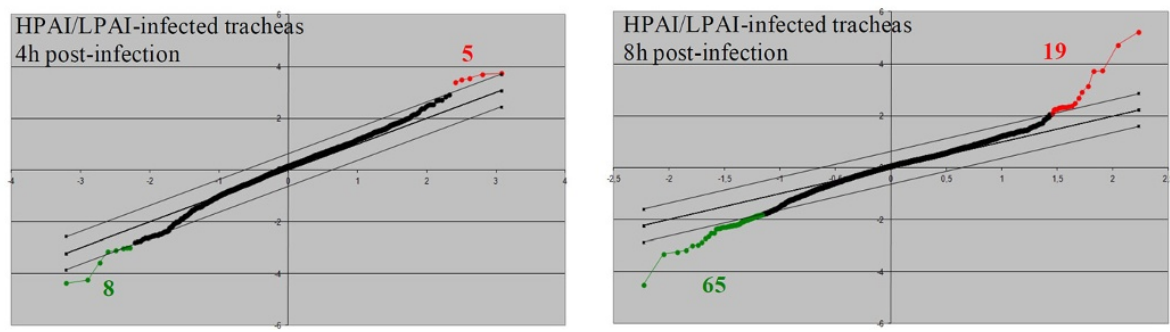

Figure 2 Plots of sequences differentially expressed in duck trachea after H5N1 infection using the significance analysis micro-array software. A and B: One-class analyses of microarray data were performed for each dataset: after LPAl-H5N1 (A) or HPAl-H5N1 (B) at $4 \mathrm{~h}$ (left panels) or $8 \mathrm{~h}$ (right panels) post-infection, as compared to mock-infected. The false discovery rate was set at 4.76 (A, left panel), $4.81(\mathbf{A}$, right panel), 5.7 (B, left panel) and 4.82\% (B, right panel). C: Two-class analyses of microarray data were performed for each time post-infection: at $4 \mathrm{~h}$ (right panel) or $8 \mathrm{~h}$ (left panel) post-infection by comparing HPAl-H5N1 infection to LPAl-H5N1 infection. The false discovery rate was set at 0 (C, right panel) and $4.15 \%$ (C, left panel). The $x$-axis values represent expected expression and the $y$-axis represent observed expression. Parallel lines represent the threshold with the corresponding false discovery rate set. Black dots represent sequences not differentially expressed between the two compared conditions. Red dots and red numbers represent significant up-regulated sequences in HPAI- or LPAI-infected tracheas as compared to mock-infected (A, B), or HPAl-infected tracheas as compared to LPAl-infected tracheas (C). Green dots and numbers represent significant down-regulated sequences in HPAl- or LPAl-infected tracheas as compared to mock-infected (A, B), or HPAl-infected tracheas as compared to LPAl-infected tracheas (C).

from the well-documented and annotated human, mouse and rat genomes.

Among the 210 genes identified from our subtracted libraries, only 158 were referenced in Ingenuity database for analysis, the remaining 52 genes are predicted genes with no relevant annotation in the database. Trachea responses, i.e. differentially expressed genes in HPAIand LPAI-infected tracheas, were compared for each time post-infection. Results are presented in Figure 3. At $4 \mathrm{~h}$ post-infection, trachea responses to infection were slightly different with 10 genes shared and 5 and 7 genes implicated only in LPAI- or HPAI-infection, respectively. Within those genes which appeared to be implicated only in LPAI- or HPAI-H5N1 infected tracheas, some genes are in fact implicated in the same protein complex (for example 20S-proteasome with PSMA2 and PSMA6). At $8 \mathrm{~h}$ post-infection, trachea responses were more different between LPAI- and HPAI-infection but some genes have potential similar functions (Additional file 1: Table S1, for example ribosomal protein L10a, L7a and LP2). Only few genes were found to be differentially expressed both at $4 \mathrm{~h}$ and at $8 \mathrm{~h}$ p.i. (7 for LPAI-infected tracheas and 9 for HPAI-infected tracheas, Figure 3).

Using the Ingenuity Pathway Analysis software, interaction networks between selected genes were inferred based on the known direct or indirect relation between these genes stored in Ingenuity's database (related to literature). In a first time the analysis was conducted on the genes obtained after comparison to mock-infected sample. For LPAI-infected tracheas, 5 gene interaction 


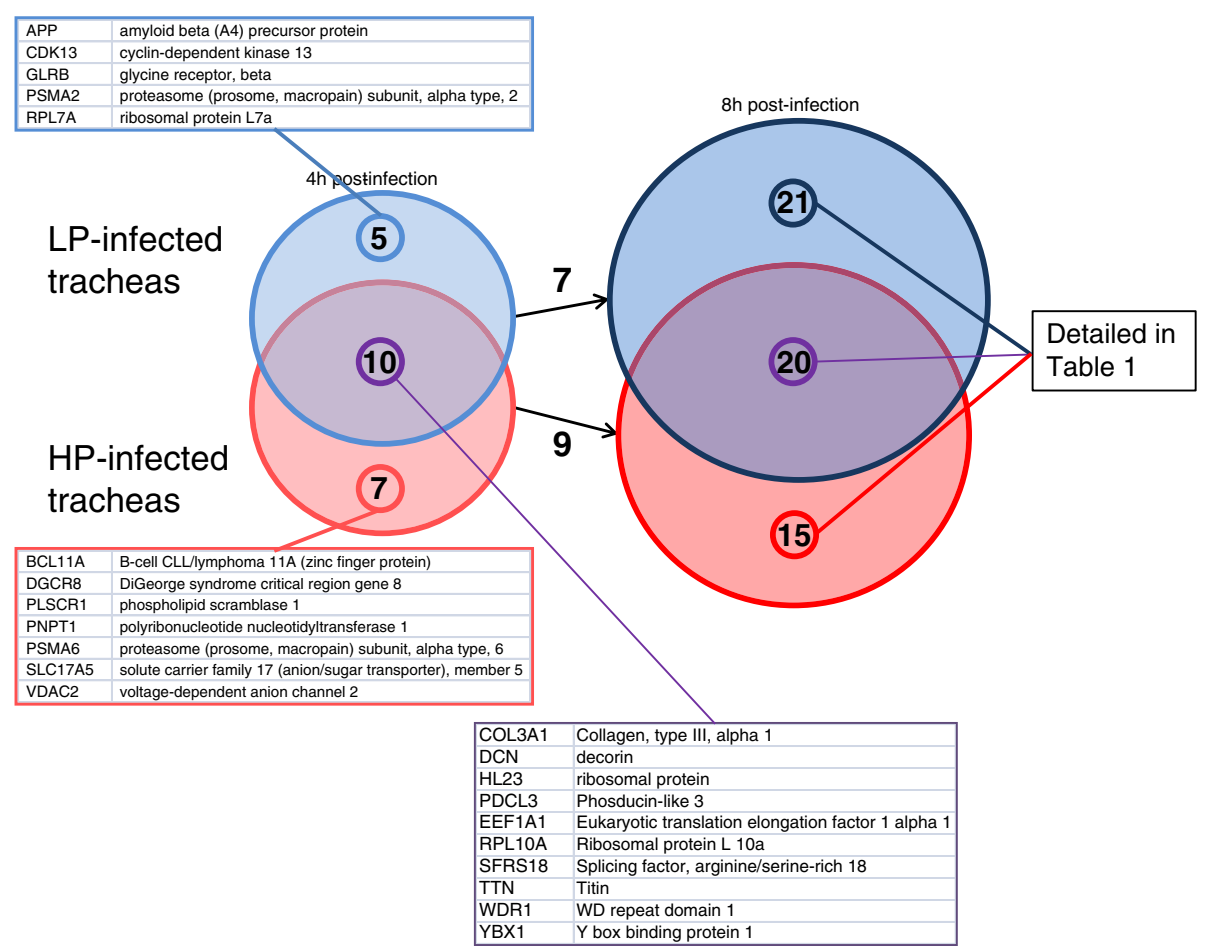

Figure 3 Schematic representation of genes significantly differentially expressed during infection time course using the ingenuity pathway analysis software. At $4 \mathrm{~h}$ post-infection (left circles) or $8 \mathrm{~h}$ post-infection (right circles), genes differentially expressed in LPAl-infected tracheas (blue circles) were compared to those differentially expressed in HPAl-infected tracheas (red circles). Common genes differentially expressed in LPAl- and HPAl-infected tracheas are represented by the junction of the two circles. Common genes differentially expressed at $4 \mathrm{~h}$ and $8 \mathrm{~h}$ post-infection are represented by the arrow between two circles. Gene lists were provided in the figure for $4 \mathrm{~h}$ post-infection and in Additional file 1: Table S1 for $8 \mathrm{~h}$ post-infection.

networks can be identified and three of these networks were connected together by one or two genes. For HPAIinfected tracheas, 5 gene interaction networks were also identified and only two of these networks interacted together by one gene, and the three others were not connected to another one. Induced and repressed genes by HPAI- or LPAI-infection included in interaction networks are presented in Additional file 1: Table S1.

Analysis of the microarray results for the signalling pathways highlighted different pathways in between LPAI- and HPAI-infected tracheas, with only the CXCR4 pathway present for both infections (Figure 4A and B). For this pathway, H-Ras, MLC and FOS were significantly modulated by both LPAI- and HPAI-infection; Rho and Gbeta were significantly modulated only by LPAI- or HPAI-infection, respectively.

In a second time, the genes selected from the comparative analysis of trachea responses after LPAI-infection or HPAI-infection were submitted to Ingenuity analysis. Only two networks, not interconnected to each other, could be identified. Repressed genes by HP-infection as compared to LP-infection included in interaction networks are presented in Additional file 2: Table S2. No induced genes were identified. Interestingly, despite the CXCR4 signalling pathway was highlighted for both HP and LP infection when compared to mock-infected samples, it was also the signalling pathway that discriminated the HP and LP infections when compared to each other. When looking at the other 9 common signalling pathways out of 10 , five were previously identified as modified into LPAIinfected trachea, and none for HPAI-infection (Figure 4C).

\section{Validation of differential expression of genes by quantitative PCR}

Differential duck tracheal gene expression after LPAI- or HPAI-infection was assessed for a selected set of genes by real-time PCRs. This set was constituted by various modulated genes i.e. induced or repressed genes either in HP or in LP infection. As shown in Table 1, quantitative PCR confirmed only for a limited number of genes the result obtained into microarray data analysis (SAM analysis) but not for the other. In particular, at $4 \mathrm{~h}$ p.i., we observed high variations in gene expression between the different samples from the same experimental condition (LPAI- or HPAI-infected tracheas) which resulted in not-statistical significant gene expression variations. Indeed, Only H-Ras, DDX3X and DCN were found statistically significantly down-regulated in HPAI- 


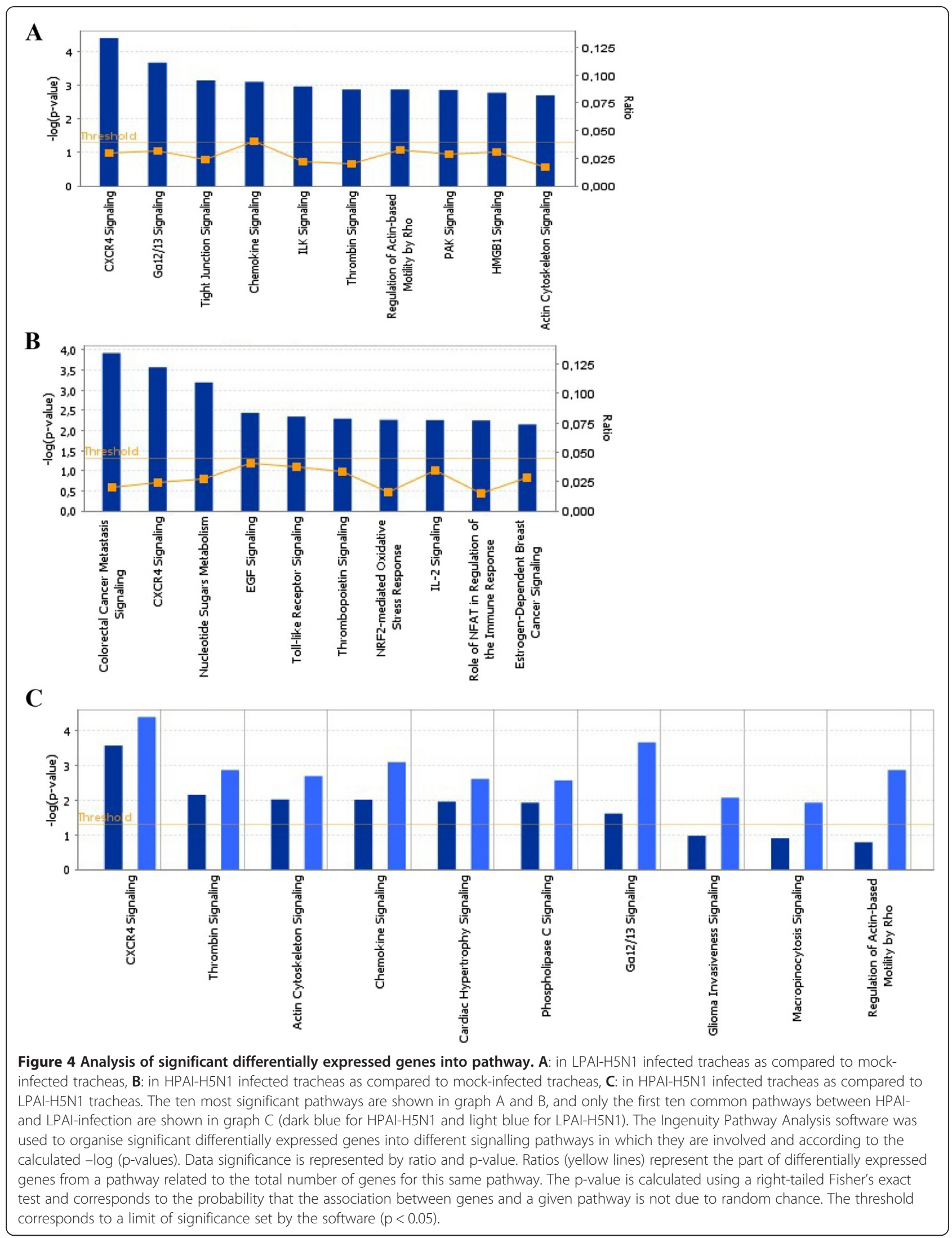


Table 1 Relative amount of differentially expressed genes in H5N1-infected tracheas as compared to control and in HPAI-H5N1- as compared to LPAI-H5N1 infected tracheas using quantitative PCR

\begin{tabular}{|c|c|c|c|c|c|c|c|}
\hline \multirow{2}{*}{$\begin{array}{l}\text { Identified } \\
\text { gene }\end{array}$} & \multirow{2}{*}{$\begin{array}{c}\text { Genbank } \\
\text { accession } \\
\text { number }\end{array}$} & \multicolumn{3}{|c|}{ Fold change at $4 \mathrm{~h}$ p.i. } & \multicolumn{3}{|c|}{ Fold change at $8 \mathrm{~h} \mathrm{p.i}$} \\
\hline & & $\mathrm{LP}$ versus control ${ }^{\mathrm{a}}$ & HP versus control ${ }^{a}$ & HP versus $L P^{b}$ & $\mathrm{LP}$ versus control ${ }^{\mathrm{a}}$ & HP versus control ${ }^{\mathrm{a}}$ & HP versus $L P^{b}$ \\
\hline TRIM33 & XM_418009 & $0.50 \pm 0.21$ & $1.41 \pm 1.34$ & 2.84 & $0.69 \pm 0.24$ & $1.19 \pm 0.15$ & $1.72^{*}$ \\
\hline H-Ras & XM_415752 & $0.87 \pm 0.47$ & $0.17 \pm 0.23$ & $0.20^{* *}$ & $0.22 \pm 0.02$ & $0.15 \pm 0.04$ & $0.68^{* *}$ \\
\hline RABL5 & CR406564 & $1.45 \pm 2.23$ & $28.85 \pm 34.10$ & 19.85 & $2.43 \pm 1.49$ & $7.06 \pm 5.30$ & 2.9 \\
\hline FOS & XM_002200534 & $3.36 \pm 2.89$ & $2.26 \pm 1.90$ & 0.67 & $0.99 \pm 0.73$ & $3.04 \pm 0.86$ & $3.08^{*}$ \\
\hline RhoA & NM_001245622 & $1.60 \pm 1.24$ & $3.96 \pm 6.14$ & 0.40 & $8.29 \pm 12.01$ & $3.89 \pm 4.41$ & 0.47 \\
\hline EEF1A1 & NM_204157 & $2.18 \pm 1.46$ & $0.15 \pm 0.03$ & 0.07 & $1.31 \pm 0.17$ & $0.59 \pm 0.25$ & $0.45^{* *}$ \\
\hline DDX3X & XM_002190542 & $1.27 \pm 0.14$ & $0.29 \pm 0.14$ & $0.22^{*}$ & $1.07 \pm 0.02$ & $0.4 \pm 0.25$ & $0.37^{* *}$ \\
\hline DCN & AF125250 & $5.95 \pm 1.94$ & $0.29 \pm 0.23$ & $0.05^{* *}$ & $0.21 \pm 0.06$ & $0.22 \pm 0.09$ & 1.02 \\
\hline IL13 & AC233977 & $13.14 \pm 12.99$ & $7.37 \pm 5.72$ & 0.56 & $5.61 \pm 3.73$ & $23.89 \pm 20.95$ & 4.26 \\
\hline
\end{tabular}

${ }^{\mathrm{a}}$ Fold change is the mean \pm SD of gene expression in 4 infected tracheas as compared to control.

${ }^{b}$ Fold change is the ratio of gene expression in HPAl-infected tracheas as compared to LPAl-infected trachea as indicated.

${ }^{*} \mathrm{p}<0.05$ and ${ }^{* *} \mathrm{p}<0.1$ (two-sample Student t-test); bold data were two-class SAM significant.

infected tracheas as compared to LPAI-infected tracheas. At $8 \mathrm{~h}$ p.i., we obtained more statistical significant results: TRIM33 and FOS were up-regulated whereas $\mathrm{H}$-Ras, EEF1A1 and DDX3X were down-regulated in HPAI-infected tracheas as compared to LPAI-infected tracheas.

\section{Discussion}

Aquatic birds play an important role into the dissemination and transmission of influenza $\mathrm{A}$ viruses between species. However, little is known concerning host/ pathogen interactions during influenza infection in these species and particularly into duck, a poorly studied species. In order to bypass the lack of information concerning duck genome, we decided the construction and use of duck trachea subtracted libraries to analyse and to compare LPAI- and HPAI-H5N1 infection.

In this study, we focused onto a primary site of infection for influenza A viruses, the trachea, for which the response to influenza infection is determining for the outcome of the infection. The in vitro model was optimised by using entire trachea instead of tracheal rings in order to limit a wound healing response that would interfere with the cellular response to infection. We further checked that the infection occurred homogeneously in the entire tracheas prepared whatever the virus used (LPAI or HPAI). M gene was detected by real-time RT-PCR and ciliostasis was observed all along the trachea for both the LPAI- and HPAI-H5N1 infected samples indicating that the virus infected and replicated all along the tracheas.

The efficacy of the SSH strategy for host response analysis and discovery of modulated genes in poorly-studied species, in association or not with microarray, has been well established [38-44]. The suppressive subtractive hybridisation $(\mathrm{SSH})$ procedure is designed to subtract cell responses that occur in both control and infected tracheas allowing focusing only on the differences between the two sets of RNA used. Even if this strategy allowed us to generate and analyse gene datasets from duck, it however suffers some drawbacks: -First, and foremost, there is an important loss of information occurring after sequencing of our subtracted libraries with only half of the sequences generating a pertinent blast result. At the end of the process only 210 different avian genes were identified. -Secondly, several sequences corresponded to genes encoding proteins with unknown function or predicted proteins that were not annotated in databases. In these conditions the annotation based analysis, with tools like the Ingenuity Pathway Analysis software, are performed with a reduced number of sequences and in fine poorly informative. Another limit of this approach resides in the fact that, due to the relative paucity of the data concerning avian species in the literature, as compared to the mammalian species, our annotation based analysis assume a functional equivalence of the avian and duck genes with their mammalian (human, rat and mouse) orthologs which is not demonstrated and most probably exaggerated. However, many signaling pathways, including pathways concerning immunity, have been described and involve the same genes in various species. Despite these limitations, our subtracted libraries corresponding to induced or repressed sequences at $4 \mathrm{~h}$ and 8 h p.i. contained 1141 and 950 clones, respectively. Using statistical analysis, only 19\% of these clones were differentially expressed. We observed that between LPAI- and HPAI-infection, there were significant differentially expressed sequences which possibly might be related to the difference of pathogenicity between LPAI and HPAI viruses. In order to identify if these sequences correspond to genes implicated in some specific mechanisms involved in influenza infection (HPAI and/or 
LPAI) in duck trachea, gene ontology annotations using Ingenuity Pathway Analysis software was used. Among identified pathways, our findings (i.e. a variation of cellular gene expression between $\mathrm{HP}$ - and LP-infected tracheas, are consistent with previous studies which demonstrate differential modulation of the immune response according to the AI strains [45-48]. Furthermore, modulation of the immune response throughout the direct interaction between cellular and viral proteins could inflect the outcome of AIV infection in the host (NS1 in particular, for a review see [49]).

In our study, the infection of duck tracheas with a HPAI- or a LPAI-H5N1 induced a different modulation of genes in the CXCR4 signalling pathway. The CXCR4 pathway is activated by the fixation of the stromal cellderived factor 1 alpha (SDF-1 $\alpha$ ) on the chemokine (CXC motif) receptor 4, a G-protein-coupled receptor. This pathway play fundamental roles in distinct signalling pathways like cell migration, transcriptional activation and cell growth, and is implicated in different physiological processes (homeostatic regulation of leukocytes traffic, haematopoiesis for example). In the case of HIV-1 infection, CXCR4 has been defined as a co-receptor which, binding by the virus, mediates membrane fusion and signalling transduction that might facilitate viral infection and pathogenesis [50]. In our case we showed that, within CXCR4 signalling pathways, G $3, \mathrm{H}-\mathrm{Ras}$, Rho, MLC and FOS expressions were modified during early time of LPAI- and/or HPAI-infection. The expression of G $\beta$ was up-regulated in HPAI-infected tracheas as compared to control. This $\mathrm{G} \beta$ protein is part of the heterotrimeric G-protein coupled to CXCR4. This activated G-protein induces transcription, cell adhesion, chemotaxis, cell survival via different signalling pathways. When comparing HPAI-infected to LPAI-infected tracheas, we found that $\mathrm{H}$-Ras was significantly more induced in LPAI-infected tracheas whereas a similar down regulation was observed for FOS. H-Ras is a small GTPase belonging to the Ras oncogene superfamily, Ras subfamily impacts multiple cellular processes (cell survival, growth, differentiation) via different pathway like the Raf/Mek/Erk pathway, PI3K pathway, or RalGDS signalling pathway and is a key intermediate in the transduction of signal during the immune response.

The FOS protein, down regulated in both HP and LP infection, is a leucine zipper protein that dimerize with c-Jun protein to form the AP-1 transcription factor complex. This complex has also been implicated in various biological processes: cell proliferation, differentiation and transformation, and apoptotic cell death. Concerning infectious diseases, FOS has been described to have a positive effect on hepatitis $\mathrm{C}$ virus ( $\mathrm{HCV}$ ) propagation and may contribute to $\mathrm{HCV}$ pathogenesis [51]. Rho was only found up-regulated in LPAI-infected tracheas. Rho family proteins are small GTPases also implicated in various cell functions, and have been described to be essential for B-lymphocytes development. These proteins, members of the CXCR4 signalling pathway, differentially expressed when comparing HPAI- and LPAI-infection, play a role in different virus infection or in immune response and might have a role in the difference of influenza virulence in ducks. Thus, it can be hypothesized that the HPAI has an inhibitory effect on the immune response in trachea by an inhibition of the CXCR4 signalling pathway, as compared to the LPAI virus, and then promotes a systemic infection in Muscovy ducks by evading the host immune response.

In our study, we also found that TRIM33 was upexpressed in HPAI-infected tracheas as compared to LPAI-infected. TRIM33, also called Trancriptional Intermediary Factor $1 \gamma$ (TIF1 $\gamma$ ), has been described to display an anti-viral activity limiting early and late gene expression in a human adenovirus infection model. This antiviral activity is counteracted by the Adenoviral E4orf3 protein which triggers TIF1 $\gamma$ to proteasomal degradation [52]. TIF1 $\gamma$ is however mainly described as transcriptional corepressor acting at the chromatin level and whether or not the up regulation of TIF1 $\gamma$ during HPAI infection is in favour of the virus or the host remains to be determined.

Dysregulation observed in this study, between these pathways and inside these pathways could explain the difference of pathogenicity between LPAI- and HPAIinfection in duck as it has been proposed by Vanderven et al. [34] and in macaques [53]. In addition, we observed a high number of differentially expressed spots corresponding to protein of unknown function or not already described which would deserve further attention in the light of host pathogen interactions.

\section{Conclusion}

In conclusion, using $\mathrm{SSH}$ and microarray tools we showed that cellular gene expression in the duck trachea was differently modulated after infection with a LPAIH5N1 or after infection with a HPAI-H5N1 virus. Some different signalling pathways and some difference within similar signalling pathways seemed to be implicated into the difference between LPAI- and HPAI-infection. These differences were found in infected trachea, a primary infection site, which could precede continuation of infection and could explain appearance of respiratory symptoms or not. Such findings have to be more precisely studied when duck genes are more characterised. Although SSH is an alternative approach to get insights into the pathogenesis of influenza infection in ducks as long as duck microarrays are not available, the lack of duck genome annotations and signalling pathways hampers understanding of host-virus interaction in this species. 


\section{Materials and methods}

Entire tracheal explants preparation, viruses and infection Entire tracheal explants were prepared from 29 days old embryonated specific pathogen free (SPF) Muscovy duck eggs (just before hatching) and after several careful washing steps, maintained at $37^{\circ} \mathrm{C}$ in minimal essential medium supplemented with antibiotics until use.

Regarding the viruses strains used, A/duck/France/ 05066b/05 (LPAI H5N1) and A/mute swan/France/ 06299/06 (HPAI H5N1 belonging to clade 2.2.1) were isolated by the National Reference Laboratory for Avian Influenza and Newcastle disease at the Anses Ploufragan/ Plouzané laboratory, France [54,55]. Influenza viruses were amplified at $37^{\circ} \mathrm{C}$ in 9-day-old SPF embryonated chicken eggs. Allantoïc fluids were collected at a maximum of 4 days after inoculation and then subjected to centrifugal clarification before use as viral stocks.

Trachea infection was performed by injecting the inoculum directly into and all along the lumen of trachea using a fine needle. Trachea was then incubated at $37^{\circ} \mathrm{C}$, $2 \% \mathrm{CO}_{2}$ in inclined plates in order to keep trachea into the medium.

\section{RNA extraction}

At 4 and $8 \mathrm{~h}$ post-infection (p.i.), tracheas were washed with cold PBS and then crushed directly into Trizol LS reagent (Invitrogen) using a tissuelyser and a stainless bead. RNA extraction was performed following standard Trizol protocol instructions.

\section{Suppressive subtraction hybridisation for libraries construction}

For libraries construction, 40 entire trachea explants were prepared and infected with a high dose $(200 \mu \mathrm{l}$ of $2 \times 10^{7}$ TCID50/ml per trachea) of the HPAI H5N1 strain (2 sets of 10 tracheas) or mock-infected (2 sets of 10 tracheas).

At 4 and $8 \mathrm{~h}$ p.i., tracheas were washed twice in cold PBS and RNA was extracted as described above. cDNA was synthesized from $4 \mu \mathrm{g}$ of each RNA preparation using the PCR-Select cDNA Subtraction Kit (Clontech). To compare the two populations of resulting cDNA (from infected cells and control cells), a suppressive subtraction hybridisation ( $\mathrm{SSH}$ ) assay was then performed using the PCR-Select cDNA Subtraction Kit (Clontech). According to manufacturer's instructions and briefly, the cDNA from the tester (infected) and from driver (mockinfected) were digested with Rsa I restriction enzyme. The tester cDNA pool was splitted into two parts and each part was then ligated to a different cDNA adaptor. During a first hybridisation step, an excess of driver (mock-infected) was added to the two tester cDNA samples, heat-denatured $\left(98^{\circ} \mathrm{C} 1 \mathrm{~min} 30\right)$ and allowed to anneal at $68^{\circ} \mathrm{C}$ during 6 to 12 hours. This step allowed for an equalization of high- and low-abundance sequences and simultaneously for a significant enrichment of differentially expressed cDNA sequences. In a second hybridisation step, the two primary hybridisation tester samples were mixed without denaturation step. To further select for differentially expressed sequences, heat-denatured driver cDNA was again added to these hybrid samples. As a result, the remaining subtracted, equalized single-stranded tester cDNA re-associated to form hybrids cDNA with a different adaptor on each end. These forward-subtracted samples were then used in PCR to amplify the differentially expressed sequences using primers complementary to adaptor. PCR mixtures of forward subtractions were ligated into pGEM vector and used in transformations with competent $E$. coli (TOP10, Invitrogen). According to Clontech's instructions, for each forward subtraction (corresponding to induced genes), reverse subtraction (corresponding to repressed genes) were also performed. These experiments resulted in the construction of four libraries: -two viralinduced libraries ( 4 and $8 \mathrm{~h}$ p.i.) and -two viral-repressed libraries ( 4 and 8 h p.i.). Libraries were conserved as bacterial clones at $-70^{\circ} \mathrm{C}$ in $\mathrm{LB} /$ glycerol medium.

\section{Sequences analysis and clone identification}

The four duck tracheal subtracted libraries were sequenced in order to identify corresponding genes. To this purpose, isolated bacterial colonies were grown for plasmid isolation with the Wizard SV 96 Plasmid DNA purification System (Promega). The DNA sequences of purified products were determined using ABI Prism DyeTerminator Cycle Sequencing Ready Reaction Kit (Applied Biosystems) and a primer flanking the cloning site of pGEM (M13rev or T7 primer) on an automatic DNA sequencer ABI 373XL (Applied Biosystems). Sequences were cleaned from vector sequences and blasted using the non redundant genbank library and also against the Gallus gallus genome (www.ncbi.nlm. nih.gov/genbank/ and www.ncbi.nlm.nih.gov/genome/ or www.ensembl.org/Gallus_gallus/). The best 5 results were considered for final identification with a maximum e-value cut-off arbitrarily set up to $2.10^{-5}$. Corresponding gene identifiers (approved symbols) were used for the annotation of the duck sequences. This heterologous annotation allowed Gene Ontology and pathway analysis using the Ingenuity Pathway Analysis data mining suite (Ingenuity Systems Inc.). Sequences were deposited into the Genbank dbEST database.

\section{Microarray production}

For microarray production, amplifications of cDNA from the 4 subtracted libraries were performed in 96-well plates using bacterial lysates. PCR amplifications were performed using a primer pair corresponding to the 
Table 2 Primer sequences used in real-time quantitative PCR

\begin{tabular}{cccc}
\hline Identified gene & Genbank accession number $^{*}$ & Primer $\mathbf{1}$ & Pimer $\mathbf{2}$ \\
\hline B-actin & EF667345 & CTTCACCACCACAGCCGAGA & TCGTGGATGCCACAGGACTC \\
DCN & AF125250 & GTGGAGATGATCCATTGATGTAAA & TGTAGCATTCAGCAAATCAAAAAT \\
DDX3X & XM_002190542 & ATATATGTCTTGTGTGCGTGTCCT & TCTAGAAATCTGTCGTGTGCAAAT \\
EEF1A1 & NM_204157 & CTTCATTAAGAACATGGTCACTGG & TCCATCTTGTTAACACCAACAATC \\
FOS & XM_002200534 & CTGGGTATCTCCAACTCGTATCTA & ACGTAAGATGGGTCATTGCTAAGT \\
H-Ras & XM_415752 & CCAGGATCCGTTGTTTCTT & ACAAACTGCGCAAGCTGAAT \\
IL13 & AC233977 & AGATAATCTGCTCCATGAGTTCC & AGCTGCTTTCCATTATTATCAGC \\
RABL5 & CR406564 & CAGTGCAGAGGACACAGAAAATC & ATAATTGACATTTCTTCCTTCTCTCT \\
RhoA & NM_001245622 & GTGTATGATTACTGGCCTITITCA & TATCCTGTGAGTGCAGAAAAGGTT \\
TRIM33 & XM_418009 & CAGCACAGGTAGCAGAGGAAG & AAGCTGCTCTCAGGACTGCTAA \\
\hline
\end{tabular}

* Accession numbers correspond to sequences showing the best blast results.

flanking adaptor sequences (Clontech) and containing a $\left(\mathrm{CH}_{2}\right)_{6}-\mathrm{NH} 2$ group at their $5^{\prime}$ end. To ensure quality and quantity amplifications, all PCR products were visualized on $1 \%$ agarose gels and then purified using Multiscreen PCR plates (Millipore). Purified products were then quantified on agarose gel and concentrations were adjusted to $200 \mathrm{ng} / \mu \mathrm{l}$. The cDNA microarray was spotted by the transcriptomic technical platform of BiogenOuest located in Nantes, France, using ROBOT and epoxysilanes coated glass slides.

\section{Probe preparation and microarray hybridisation}

For probe preparation, 96 entire trachea explants were prepared and separated into three groups. Two groups were infected with either a high dose $\left(200 \mu \mathrm{l}\right.$ of $2 \times 10^{7}$ TCID $50 / \mathrm{ml}$ per trachea) of the HPAI H5N1 strain (32 tracheas) or a high dose ( $200 \mu \mathrm{l}$ of $2 \times 10^{7} \mathrm{TCID} 50 / \mathrm{ml}$ per trachea) of the LPAI H5N1 strain (32 tracheas). A third group of 32 tracheas was mock-infected (control). Within each groups (infected or not) tracheas were treated individually. At 4 and 8 h p.i., RNA was extracted from 16 out of 32 of each set of tracheas as described in 2.2. RNA was quantified using the Qubit quantitation platform (Invitrogen). For RNA from infected tracheas, four random pools were constructed each with 4 individual RNAs whereas for RNA from mock-infected tracheas only one single reference pool (16 individual RNA) was created to ensure an homogeneous baseline between the different experimental conditions. Quality of RNA in each pool was checked using the Bioanalyzer 2100 platform (Agilent). Probes synthesis was performed with $500 \mathrm{ng}$ of pooled RNA and using the Amino Allyl MessageAmp ${ }^{\mathrm{Tm}}$ II aRNA Amplification kit (Ambion) and CyDye (Cy3/Cy5) Reactive Dye Pack (Amersham). Two rounds of amplification and a quantity limitation for the $2^{\text {nd }}$ round in order to increase the production of labelled products were performed. Dye swap Cy3/Cy5 was performed between infected and mock-infected samples. Each Cy3- (or Cy5-) labelled sample from infected tracheas was then hybridised with the Cy5- (or Cy3-) labelled reference sample from mock-infected tracheas on our microarray slide. Hybridisations were performed overnight at $42^{\circ} \mathrm{C}$ in Arraylt hybridisation chambers (Telechem). Images of the hybridised arrays were acquired by scanning using a Genepix 4000A scanner with the GenePix Pro 5.0 data acquisition and analysis software (Axon Instruments).

\section{Microarray analysis}

Microarray data were processed as previously described $[17,56]$. Briefly, the raw data were normalised (Lowess) then subjected to a statistical analysis using the significance analysis of microarray (SAM) software to identify differentially expressed genes [57]. SAM software also calculated a false discovery rate (FDR) for each analysis performed. In a first analysis, results obtained with HPAI- or LPAI-infected tracheas were compared to those obtained with mock-infected tracheas at $4 \mathrm{~h}$ or $8 \mathrm{~h}$ p.i. (SAM one-class analyses). In a second analysis, results obtained with HPAI-infected tracheas were compared to those obtained with LPAI-infected tracheas (SAM two-class analyses). These analyses resulted in a total of 6 datasets (HPAI versus test at 4 and $8 \mathrm{~h}$ pi, LPAI versus test at 4 and $8 \mathrm{~h}$ pi, HPAI versus LPAI at 4 and $8 \mathrm{~h} \mathrm{pi)}$ of genes differentially expressed from our different experimental conditions. Results of microarray, SAM analysis and sequencing were combined and used in order to identify implicated cellular pathways by Gene Ontology using the Ingenuity Pathway Analysis software (Ingenuity Systems Inc.). Due to the relative poverty on duck and chicken data in the databases, as compared to the human, mouse or rat data, those analyses were performed by homology with human or mouse genes.

\section{Real-time PCRs}

For the validation of the experimental infection model, in vitro infection of entire trachea, a standard 
amplification of influenza matrix (M) segment using real-time RT-PCR method was performed [58].

Relative amount of 10 genes of interest as well as control (beta-actin) house-keeping gene expression in HPAI-, LPAI- or mock-infected duck tracheas was analysed by real-time PCR. Each individual RNA sample (see section 2.6) was reverse transcribed using a polydT primer and Superscript II reverse transcriptase (Invitrogen) according to the manufacturer's protocol. cDNA was subjected to real-time PCR using gene-specific primers (Table 2) and SYBR green technology. Real-time PCR reactions were performed in a final volume of $25 \mu \mathrm{l}$ with $12.5 \mu \mathrm{l}$ of SYBR Green PCR Master Mix (Applied Biosystems), $10 \mu \mathrm{l}$ of cDNA (diluted 1:10) and $200 \mathrm{nM}$ of each forward and reverse primer. Amplifications were performed using the following conditions: $2 \mathrm{~min}$ at $50^{\circ} \mathrm{C}, 10 \mathrm{~min}$ at $95^{\circ} \mathrm{C}$, followed by 45 amplification cycles $\left(15 \mathrm{sec}\right.$ at $95^{\circ} \mathrm{C}$ and $1 \mathrm{~min}$ at $60^{\circ} \mathrm{C}$ ). The uniqueness of the amplification product was assessed by a dissociation stage after the last amplification cycle. Amplifications were carried out in 96-well plates with 3 replicates per sample, using Applied Biosystem 7500 Real-Time PCR Systems and analysed with the Sequence Detection Software. Results were normalized by the control house-keeping gene expression in each sample. Relative amounts of each gene expression were calculated using the $R=2^{-\Delta \Delta C T}$ equation [59] with gene expression in mock-infected tracheas as relative reference. Two-sample Student t-test was performed to compare gene expression variation between HPAI- and LPAI-infected tracheas, using Systat 9 software (Systat Software Inc., Point Richmond, CA, USA).

\section{Additional files}

Additional file 1: Table S1. Ingenuity annotated responding genes to HPAl- or LPAl-infection as compared to control at $8 \mathrm{~h}$ post-infection.

Additional file 2: Table S2. Ingenuity annotated responding genes to HPAl-infection as compared to LPAl-infection at $8 \mathrm{~h}$ post-infection.

\section{Competing interests}

The authors declare that they have no conflict of interest.

\section{Authors' contributions}

PM and Véronique Jestin created the original idea of this research and designed the study. PM conducted assays and analysis, data interpretation and drafted the manuscript. $\mathrm{CD}, \mathrm{AO}$ and $\mathrm{HQ}$ performed laboratory methods and preliminary analysis. FXB performed a part of bioinformatics analysis. YB performed statistical analysis, and contributed to the manuscript writing. All authors read and approved the final manuscript.

\section{Acknowledgements}

This work was supported by EU $6^{\text {th }}$ framework Flupath ( $n^{\circ}$ 044220). The authors would like to thank Dave Burt and Eric Niqueux for critical discussion about the methods to analyse data, Véronique Jestin for critical reading of the manuscript and Paul Brown for English corrections.

\section{Author details}

${ }^{1}$ Anses Ploufragan/Plouzané laboratory, Virologie Immunologie Parasitologie Aviaires et Cunicoles, B.P. 53, Ploufragan 22440, France. 'Anses Ploufragan/
Plouzané laboratory, Génétique Virale et Biosécurité, B.P. 53, Ploufragan 22440, France. ${ }^{3}$ European University of Brittany, Rennes, France.

Received: 9 January 2013 Accepted: 6 September 2013

Published: 10 September 2013

\section{References}

1. Palese $P$, Shaw ML: Orthomyxoviridae : the viruses and their replication. In Fields virology. 5th edition. Edited by Knipe PMH DM, Griffin DE, Lamb RA, Martin MA, Roizman B, Straus SE. Philadelphia: Lippincott-Raven; 2007:1647-1689.

2. Rott R: The pathogenic determinant of influenza virus [published erratum appears in Vet Microbiol Apr; 34(4):398]. Vet Microbiol 1992, 33:303-310

3. Pantin-Jackwood MJ, Swayne DE: Pathogenesis and pathobiology of avian influenza virus infection in birds. Rev Sci Tech 2009, 28:113-136.

4. FAO-OIE-WHO: H5N1 avian influenza: Timeline of major events. Influenza/ Human animal interface tripartite note; 2011. www.who.int.

5. OIE: Outbreaks of Highly Pathogenic Avian Influenza (subtype H5N1) in poultry from the end of 2003 to 12 December 2011. Animal health in the world; 2011. wWw.oie.int.

6. WHO: H5N1 avian influenza: Timeline of major events. H5N1 avian influenza: 2011. www.who.int.

7. WHO: Cumulative number of confirmed human cases for avian influenza $A$ (H5N1) reported to WHO, 2003-2013. H5N1 cumulative table; 2013. www.who.int.

8. Coleman JR: The PB1-F2 protein of Influenza A virus: increasing pathogenicity by disrupting alveolar macrophages. Virol J 2007, 4:9.

9. Garcia-Sastre A, Durbin RK, Zheng H, Palese P, Gertner R, Levy DE, Durbin JE: The role of interferon in influenza virus tissue tropism. J Virol 1998, 72:8550-8558

10. Heynisch B, Frensing T, Heinze K, Seitz C, Genzel Y, Reichl U: Differential activation of host cell signalling pathways through infection with two variants of influenza A/Puerto Rico/8/34 (H1N1) in MDCK cells. Vaccine 2010, 28:8210-8218.

11. Talon J, Horvath CM, Polley R, Basler CF, Muster T, Palese P, Garcia-Sastre A Activation of interferon regulatory factor 3 is inhibited by the influenza A virus NS1 protein. J Virol 2000, 74:7989-7996.

12. Watanabe T, Watanabe $S$, Kawaoka Y: Cellular networks involved in the influenza virus life cycle. Cell Host Microbe 2010, 7:427-439.

13. Zamarin D, Garcia-Sastre A, Xiao X, Wang R, Palese P: Influenza virus PB1-F2 protein induces cell death through mitochondrial ANT3 and VDAC1. PLoS Pathog 2005, 1:e4

14. Alkhalil A, Hammamieh R, Hardick J, Ichou MA, Jett M, Ibrahim S: Gene expression profiling of monkeypox virus-infected cells reveals novel interfaces for host-virus interactions. Virol J 2010, 7:173.

15. Almeida PE, Weber PS, Burton JL, Tempelman RJ, Steibel JP, Zanella AJ: Gene expression profiling of peripheral mononuclear cells in lame dairy cows with foot lesions. Vet Immunol Immunopathol 2007, 120:234-245.

16. Mo XY, Ma W, Zhang Y, Zhao H, Deng Y, Yuan W, Wang Y, Li Y, Zhu C, Liu M, Wu X: Microarray analyses of differentially expressed human genes and biological processes in ECV304 cells infected with rubella virus. J Med Virol 2007, 79:1783-1791.

17. Renson P, Blanchard Y, Le-Dimna M, Felix H, Cariolet R, Jestin A, Le-Potier MF: Acute induction of cell death-related IFN stimulated genes (ISG) differentiates highly from moderately virulent CSFV strains. Vet Res 2010, 41:7.

18. Tong HH, Long JP, Li D, DeMaria TF: Alteration of gene expression in human middle ear epithelial cells induced by influenza $A$ virus and its implication for the pathogenesis of otitis media. Microb Pathog 2004, 37:193-204

19. Yu C, Boon D, McDonald SL, Myers TG, Tomioka K, Nguyen H, Engle RE, Govindarajan S, Emerson SU, Purcell RH: Pathogenesis of hepatitis E virus and hepatitis $C$ virus in chimpanzees: similarities and differences. J Virol 2010, 84:11264-11278.

20. Zornetzer GA, Frieman MB, Rosenzweig E, Korth MJ, Page C, Baric RS, Katze MG: Transcriptomic analysis reveals a mechanism for a prefibrotic phenotype in STAT1 knockout mice during severe acute respiratory syndrome coronavirus infection. J Virol 2010, 84:11297-11309.

21. Suzuki K, Okada H, Itoh T, Tada T, Mase M, Nakamura K, Kubo M, Tsukamoto K: Association of increased pathogenicity of Asian H5N1 
highly pathogenic avian influenza viruses in chickens with highly efficient viral replication accompanied by early destruction of innate immune responses. J Virol 2009, 83:7475-7486.

22. Wang Y, Brahmakshatriya V, Zhu H, Lupiani B, Reddy SM, Yoon BJ, Gunaratne PH, Kim JH, Chen R, Wang J, Zhou H: Identification of differentially expressed miRNAs in chicken lung and trachea with avian influenza virus infection by a deep sequencing approach. BMC Genomics 2009, 10:512.

23. Xing Z, Cardona CJ, Li J, Dao N, Tran T, Andrada J: Modulation of the immune responses in chickens by low-pathogenicity avian influenza virus H9N2. J Gen Virol 2008, 89:1288-1299.

24. Dar A, Munir S, Vishwanathan S, Manuja A, Griebel P, Tikoo S, Townsend H, Potter A, Kapur V, Babiuk LA: Transcriptional analysis of avian embryonic tissues following infection with avian infectious bronchitis virus. Virus Res 2005, 110:41-55.

25. Li X, Chiang HI, Zhu J, Dowd SE, Zhou H: Characterization of a newly developed chicken 44K Agilent microarray. BMC Genomics 2008, 9:60.

26. Munir S, Sharma JM, Kapur V: Transcriptional response of avian cells to infection with Newcastle disease virus. Virus Res 2005, 107:103-108.

27. Reemers SS, Groot Koerkamp MJ, Holstege FC, Van-Eden W, Vervelde L: Cellular host transcriptional responses to influenza $A$ virus in chicken tracheal organ cultures differ from responses in in vivo infected trachea. Vet Immunol Immunopathol 2009, 132:91-100.

28. Reemers SS, Van-Leenen D, Koerkamp MJ, Van-Haarlem D, van de-Haar $P$, Van-Eden W, Vervelde L: Early host responses to avian influenza A virus are prolonged and enhanced at transcriptional level depending on maturation of the immune system. Mol Immunol 2010, 47:1675-1685.

29. Sarmento L, Afonso CL, Estevez C, Wasilenko J, Pantin-Jackwood M: Differential host gene expression in cells infected with highly pathogenic H5N1 avian influenza viruses. Vet Immunol Immunopathol 2008, 125:291-302.

30. Zhang W, Li H, Cheng G, Hu S, Li Z, Bi D: Avian influenza virus infection induces differential expression of genes in chicken kidney. Res Vet Sci 2008, 84:374-381.

31. Huang YH, Li N, Burt DW, Wu F: Genomic research and applications in the duck (Anas platyrhynchos). Worlds Poult Sci I 2008, 64:329-341.

32. Huang Y, Li Y, Burt DW, Chen H, Zhang Y, Qian W, Kim H, Gan S, Zhao Y, Li J, et al: The duck genome and transcriptome provide insight into an avian influenza virus reservoir species. Nat Genet 2013, 45:776-783.

33. Fleming-Canepa X, Brusnyk C, Aldridge JR, Ross KL, Moon D, Wang D, Xia J, Barber MR, Webster RG, Magor KE: Expression of duck CCL19 and CCL21 and CCR7 receptor in lymphoid and influenza-infected tissues. Mol Immunol 2011, 48:1950-1957.

34. Vanderven HA, Petkau K, Ryan-Jean KE, Aldridge JR Jr, Webster RG, Magor KE: Avian influenza rapidly induces antiviral genes in duck lung and intestine. Mol Immunol 2012, 51:316-324.

35. Adams SC, Xing Z, Li J, Cardona CJ: Immune-related gene expression in response to $\mathrm{H} 11 \mathrm{~N} 9$ low pathogenic avian influenza virus infection in chicken and Pekin duck peripheral blood mononuclear cells. Mol Immunol 2009, 46:1744-1749.

36. Cagle C, To TL, Nguyen T, Wasilenko J, Adams SC, Cardona CJ, Spackman E, Suarez DL, Pantin-Jackwood MJ: Pekin and Muscovy ducks respond differently to vaccination with a H5N1 highly pathogenic avian influenza (HPAl) commercial inactivated vaccine. Vaccine 2011, 29:6549-6557.

37. Phuong do Q, Dung NT, Jorgensen PH, Handberg K, Vinh NT, Christensen JP: Susceptibility of Muscovy (Cairina Moschata) and mallard ducks (Anas Platyrhynchos) to experimental infections by different genotypes of H5N1 avian influenza viruses. Vet Microbiol 2010, 148:168-174.

38. Dios S, Poisa-Beiro L, Figueras A, Novoa B: Suppression subtraction hybridization (SSH) and macroarray techniques reveal differential gene expression profiles in brain of sea bream infected with nodavirus. Mol Immunol 2007, 44:2195-2204

39. Fang Q, Wang L, Zhu J, Li Y, Song Q, Stanley DW, Akhtar ZR, Ye G Expression of immune-response genes in lepidopteran host is suppressed by venom from an endoparasitoid. Pteromalus puparum. BMC Genomics 2010, 11:484.

40. Hu TX, Yu M, Zhao J: Comparative transcriptional profiling analysis of the two daughter cells from tobacco zygote reveals the transcriptome differences in the apical and basal cells. BMC Plant Biol 2010, 10:167.
41. Munir S, Singh S, Kaur K, Kapur V: Suppression subtractive hybridization coupled with microarray analysis to examine differential expression of genes in virus infected cells. Biol Proced Online 2004, 6:94-104.

42. Lake J, Gravel C, Koko GK, Robert C, Vandenberg GW: Combining suppressive subtractive hybridization and CDNA microarrays to identify dietary phosphorus-responsive genes of the rainbow trout (Oncorhynchus mykiss) kidney. Comp Biochem Physiol Part D Genomics Proteomics 2010, 5:24-35.

43. Pan YS, Lee YS, Lee YL, Lee WC, Hsieh SY: Differentially profiling the low-expression transcriptomes of human hepatoma using a novel $\mathrm{SSH} /$ microarray approach. BMC Genomics 2006, 7:131.

44. Zou X, Jiang Y, Liu L, Zhang Z, Zheng Y: Identification of transcriptome induced in roots of maize seedlings at the late stage of waterlogging BMC Plant Biol 2010, 10:189.

45. Hsu AC, Barr I, Hansbro PM, Wark PA: Human influenza is more effective than avian influenza at antiviral suppression in airway cells. Am J Respir Cell Mol Biol 2010, 44:906-913.

46. Iwai A, Shiozaki T, Kawai T, Akira S, Kawaoka Y, Takada A, Kida H, Miyazaki T: Influenza A virus polymerase inhibits type I interferon induction by binding to interferon beta promoter stimulator 1. J Biol Chem 2010, 285:32064-32074

47. Liu H, Golebiewski L, Dow EC, Krug RM, Javier RT, Rice AP: The ESEV PDZ-binding motif of the avian influenza A virus NS1 protein protects infected cells from apoptosis by directly targeting Scribble. J Virol 2010, 84:11164-11174

48. Wang S, Quang Le T, Chida J, Cisse Y, Yano M, Kido H: Mechanisms of matrix metalloproteinase- 9 upregulation and tissue destruction in various organs in influenza A virus infection. J Med Invest 2010, 57:26-34.

49. Hale BG, Randall RE, Ortin J, Jackson D: The multifunctional NS1 protein of influenza A viruses. J Gen Virol 2008, 89:2359-2376.

50. Wu Y, Yoder A: Chemokine coreceptor signaling in HIV-1 infection and pathogenesis. PLoS Pathog 2009, 5:e1000520.

51. Kang SM, Lim S, Won SJ, Shin YJ, Lim YS, Ahn BY, Hwang SB: c-Fos regulates hepatitis $C$ virus propagation. FEBS Lett 2011, 585:3236-3244.

52. Forrester NA, Patel RN, Speiseder T, Groitl P, Sedgwick GG, Shimwell NJ, Seed RI, Catnaigh PO, McCabe CJ, Stewart GS, et al: Adenovirus E4orf3 targets transcriptional intermediary factor 1gamma for proteasome-dependent degradation during infection. J Virol 2012, 86:3167-3179.

53. Cilloniz C, Shinya K, Peng X, Korth MJ, Proll SC, Aicher LD, Carter VS, Chang JH, Kobasa D, Feldmann F, et al: Lethal influenza virus infection in macaques is associated with early dysregulation of inflammatory related genes. PLoS Pathog 2009, 5:e1000604.

54. Briand FX, Le-Gall-Reculé G, Guillou-Cloarec C, Ogor K, Jestin V: Phylogeny and genotyping of recent avian low-pathogenic $\mathrm{H} 5$ subtype influenza viruses from French ducks. J Gen Virol 2010, 91:960-970.

55. Gall-Recule GL, Briand FX, Schmitz A, Guionie O, Massin P, Jestin V: Double introduction of highly pathogenic $\mathrm{H} 5 \mathrm{~N} 1$ avian influenza virus into France in early 2006. Avian Pathol 2008, 37:15-23.

56. Blanchard Y, Le-Meur N, Le-Cunff M, Blanchard P, Leger J, Jestin A: Cellular gene expression survey of PseudoRabies Virus (PRV) infected Human Embryonic Kidney cells (HEK-293). Vet Res 2006, 37:705-723.

57. Tusher VG, Tibshirani R, Chu G: Significance analysis of microarrays applied to the ionizing radiation response. Proc Natl Acad Sci USA 2001, 98:5116-5121.

58. Spackman E, Senne DA, Myers TJ, Bulaga LL, Garber LP, Perdue ML, Lohman $K$, Daum LT, Suarez DL: Development of a real-time reverse transcriptase $\mathrm{PCR}$ assay for type $\mathrm{A}$ influenza virus and the avian $\mathrm{H} 5$ and $\mathrm{H} 7$ hemagglutinin subtypes. J Clin Microbiol 2002, 40:3256-3260.

59. Livak KJ, Schmittgen TD: Analysis of relative gene expression data using real-time quantitative PCR and the 2(-Delta Delta $C(T))$ Method. Methods 2001, 25:402-408.

doi:10.1186/1743-422X-10-279

Cite this article as: Massin et al.: Differential cellular gene expression in duck trachea infected with a highly or low pathogenic H5N1 avian influenza virus. Virology Journal 2013 10:279. 